\title{
ARTICLE
}

Translational Therapeutics

\section{Complementary activity of tyrosine kinase inhibitors against secondary kit mutations in imatinib-resistant gastrointestinal stromal tumours}

\author{
César Serrano ${ }^{1,2,3}$, Adrián Mariño-Enríquez ${ }^{1}$, Derrick L. Tao ${ }^{1}$, Julia Ketzer ${ }^{4}$, Grant Eilers ${ }^{1}$, Meijun Zhu ${ }^{1}$, Channing Yu ${ }^{2,5,11}$, \\ Aristotle M. Mannan ${ }^{5}$, Brian P. Rubin ${ }^{6}$, George D. Demetri ${ }^{2,7}$, Chandrajit P. Raut ${ }^{2,8}$, Ajia Presnell $^{9}$, Arin McKinley ${ }^{9}$, Michael C. Heinrich $^{9}{ }^{9}$, \\ Jeffrey T. Czaplinski ${ }^{2}$, Ewa Sicinska ${ }^{10}$, Sebastian Bauer ${ }^{4}$, Suzanne George ${ }^{2}$ and Jonathan A. Fletcher ${ }^{1,2}$
}

\begin{abstract}
BACKGROUND: Most patients with KIT-mutant gastrointestinal stromal tumours (GISTs) benefit from imatinib, but treatment resistance results from outgrowth of heterogeneous subclones with KIT secondary mutations. Once resistance emerges, targeting KIT with tyrosine kinase inhibitors (TKIs) sunitinib and regorafenib provides clinical benefit, albeit of limited duration. METHODS: We systematically explored GIST resistance mechanisms to KIT-inhibitor TKIs that are either approved or under investigation in clinical trials: the studies draw upon GIST models and clinical trial correlative science. We subsequently modelled in vitro a rapid TKI alternation approach against subclonal heterogeneity.

RESULTS: Each of the KIT-inhibitor TKIs targets effectively only a subset of KIT secondary mutations in GIST. Regorafenib and sunitinib have complementary activity in that regorafenib primarily inhibits imatinib-resistance mutations in the activation loop, whereas sunitinib inhibits imatinib-resistance mutations in the ATP-binding pocket. We find that rapid alternation of sunitinib and regorafenib suppresses growth of polyclonal imatinib-resistant GIST more effectively than either agent as monotherapy.

CONCLUSIONS: Our data highlight that heterogeneity of KIT secondary mutations is the main mechanism of tumour progression to KIT inhibitors in imatinib-resistant GIST patients. Therapeutic combinations of TKIs with complementary activity against resistant mutations may be useful to suppress growth of polyclonal imatinib-resistance in GIST.
\end{abstract}

British Journal of Cancer (2019) 120:612-620; https://doi.org/10.1038/s41416-019-0389-6

\section{BACKGROUND}

Gastrointestinal stromal tumour (GIST) is a mesenchymal tumour of the gastrointestinal tract and the most common subtype of human sarcoma. ${ }^{1,2}$ KIT or PDGFRA receptor tyrosine kinase gainof-function mutations are crucial initiating oncogenic events in $90 \%$ of GISTs, $^{3,4}$ resulting in oncogenic addiction. Therefore, abolition of KIT or PDGFRA signalling with tyrosine kinase inhibitors (TKIs) profoundly impairs GIST cell viability and growth. ${ }^{5,6}$

First-line imatinib mesylate (Gleevec, Novartis Oncology, Basel, Switzerland) inhibits activity of mutant KIT and PDGFRA, and substantially improves survival in most GIST patients. ${ }^{6,7}$ However, most patients with initial clinical benefit from imatinib eventually progress, typically in 20-24 months. ${ }^{6,7}$ Oncogenically-activated KIT continues to be the key driver of GIST proliferation and survival after imatinib failure in up to $90 \%$ of the patients, due to reactivation of KIT signalling by tumour subclones with heterogeneous secondary KIT mutations. ${ }^{8-11}$ These KIT secondary mutations cluster in two regions of the kinase domain: the ATPbinding pocket (encoded by exons 13 and 14) and the activation loop (encoded by exons 17 and 18).

Current drug development strategies for imatinib-resistant GIST exploit continued KIT-dependency by seeking TKIs which inhibit a broader spectrum of KIT secondary mutations. These efforts led to regulatory approval of sunitinib (Sutent, Pfizer Inc.; New York, USA) and regorafenib (Stivarga, Bayer HealthCare Pharmaceuticals Inc., Montville, NJ, USA) as second- and third-line therapies, respectively, for patients with advanced GIST. ${ }^{12-15}$ Other multikinase inhibitors are currently in phase I to phase III clinical studies. ${ }^{16,17}$ Because sunitinib and regorafenib are broadly-active multi-kinase

\footnotetext{
${ }^{1}$ Department of Pathology, Brigham and Women's Hospital, Harvard Medical School, 20 Shattuck Street, Thorn 528, Boston, MA, USA; ${ }^{2}$ Department of Medical Oncology, Dana-

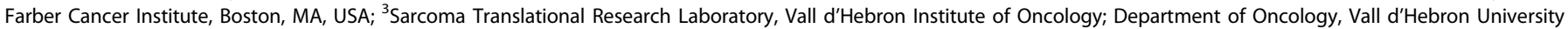

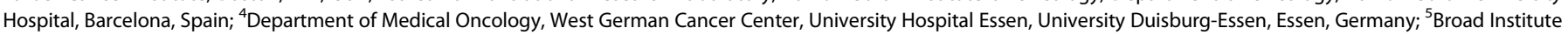

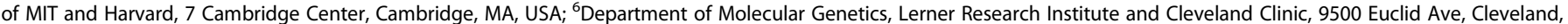

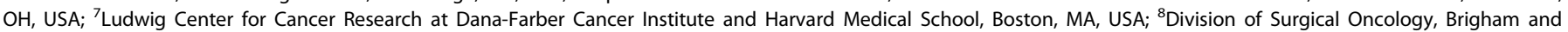

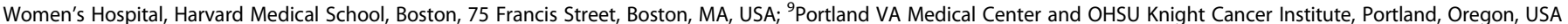
and ${ }^{10}$ Department of Oncologic Pathology, Dana Farber Cancer Institute, Boston, MA, USA

Correspondence: César Serrano (cserrano@vhio.net) or Jonathan A. Fletcher (jfletcher@partners.org)

${ }^{11}$ Present address: Daiichi Sankyo Inc., Basking Ridge, NJ, USA

These authors contributed equally: Suzanne George, Jonathan A. Fletcher.
}

Received: 17 July 2018 Revised: 28 September 2018 Accepted: 22 October 2018

Published online: 22 February 2019 
inhibitors, they are associated with more toxicities than imatinib in most GIST patients. Further, after clinical progression of advanced GIST on imatinib, the clinical benefit of sunitinib and regorafenib is limited, with median time to progression of 6 months or less. ${ }^{16,17}$ Our previous studies have shown that some but not all imatinibresistant KIT-mutations respond to sunitinib. Specifically, in vitro and clinical studies demonstrated that sunitinib is active against imatinib-resistant GIST subclones with KIT ATP-binding pocket V654A secondary mutations, but inactive against subclones with KIT activation loop mutations. ${ }^{12,18}$ Thus, clinical progression on sunitinib occurs after a median time of 6 months, ${ }^{14}$ mainly due to the emergence of cross-resistant KIT-dependent subclones. ${ }^{8-11}$ Likewise, polyclonal heterogeneity of KIT-driven, imatinib-resistant subclones might also lead to the modest benefit observed with regorafenib and other available TKIs as single-agent therapies.

In this study we further explored the relationship between secondary KIT kinase mutations and the activity of third-line regorafenib and other TKIs with KIT inhibitory activity being investigated in GIST clinical trials. To this end, we determined the activity of multiple TKIs against imatinib-sensitive and imatinibresistant KIT mutations in clinically representative, patient-derived GIST models. We found that regorafenib, as well as all small molecule KIT-inhibitor monotherapies, have a drug-specific activity profile against a subset of the KIT secondary mutational spectrum. Interestingly, some of these TKIs, including second- and third-line sunitinib and regorafenib, have complementary activity against resistant clones, and therefore we further undertook an in vitro proof-of-concept combination strategy based on rapid TKI alternation aiming to deter growth of polyclonal imatinib-resistant subclones.

\section{MATERIALS AND METHODS}

Cell culture studies

$\mathrm{CHO}$ cells were from the American Type Culture Collection (Manassus, VA, USA) and were transfected with KIT-mutant cDNA constructs as previously described ${ }^{9}$ and treated with imatinib, sunitinib or regorafenib. GIST cells used in these studies were derived from human GIST metastases and have been published previously ${ }^{19}$ with the exception of GIST226, which is a novel KITnegative GIST line that contains (but does not express) homozygous primary KIT exon 11 in-frame deletion (P551-W557) and homozygous KIT exon 17 Y823D mutations. All lines were credentialed by Sanger sequencing evaluations of known mutations, at baseline and every 3 months during the study. All cultures were shown to be mycoplasma-free.

\section{Protein blotting}

Whole cell lysates were prepared as described previously, ${ }^{20}$ and protein concentrations were determined using the Bio-Rad protein assay (Bio-Rad, Hercules, CA, USA). KIT immunoprecipitations, in the $\mathrm{CHO}$ cell assays, were as described previously. ${ }^{9}$ Electrophoresis, immunoblotting, and chemiluminescence detection were as described previously. ${ }^{21}$ Primary antibodies to phospho-KIT Y721 (\#3391), phospho-KIT Y703 (\#3073), phosphoAKT S473 (\#9271), AKT (\#9272), phospho-RB1 S795 (\#9301) and RB1 (\#9309) were from Cell Signaling Technology (Danvers, MA, USA); to KIT (\#A4502) were from Dako (Carpinteria, CA, USA); to actin (\#A4700) were from Sigma (San Luis, MI, USA); and to Cyclin A (clone 6E6) were from Leica Byosistems (Wetzlar, Germany).

\section{Immunohistochemistry}

Immunohistochemical staining for Ki-67 was performed against cell cultures on chamber slides with an antibody (\#0505) from Immunotech (Marseille, France) at dilution of 1:200. Then the slides were incubated with a biotin-conjugated secondary antibody and stained using the Ventana (Tucson, AZ, USA) DAB detection kit with counterstaining by haematoxylin.
Reagents

Ponatinib and regorafenib were from Selleck Chemicals (Houston, TX, USA). Dovitinib, dasatinib, imatinib, masitinib, nilotinib, sunitinib, and sorafenib were from LC Laboratories (Woburn, MA, USA).

\section{Cell viability studies}

The sulforhodamine B (SRB) assay was used according to the method of Skehan. ${ }^{22}$ Cells were plated in 96-well flat-bottomed plates. After $24 \mathrm{~h}$ culture medium was replaced with fresh medium (with or without drugs) in triplicate cultures. At the end of drug exposure $(72 \mathrm{~h})$, cells were fixed for $1 \mathrm{~h}$ and stained with $0.4 \%$ SRB (Sigma Aldrich, St. Louis, MO USA) and the optical density was detected at $560 \mathrm{~nm}$. Each experiment was repeated three times.

\section{Clinical correlative studies}

Tumour specimens for genotype analyses were obtained from patients enrolled on a phase II clinical trial of regorafenib in GIST. ${ }^{23}$ Briefly, patients were adults who had histologically confirmed metastatic and/or unresectable GIST with progression or intolerance to imatinib and prior failure to sunitinib. Tumour tissue was analysed in patients receiving regorafenib $160 \mathrm{mg}$ daily 3-weeks on, 1-week off. Objective response was assessed by computed tomography (CT) in genotyped patients at baseline and at the end of every even-numbered cycle. Disease status was assessed using Response Evaluation Criteria in Solid Tumours (RECIST) as complete response (CR), partial response (PR), stable disease (SD), or progressive disease (PD). ${ }^{24}$ Metabolic response was assessed by serial $\left[{ }^{18} \mathrm{~F}\right]$ fluoro-2-deoxy-D-glucose positron emission tomography (FDG-PET) scans were done in a fasting state $1 \mathrm{~h}$ following i.v. administration of FDG $(15-20 \mathrm{mCi})$ at baseline, at the end of cycle 1 and cycle 4 dosing.

GIST xenograft studies

A patient-derived xenograft (PDX) model, PG48, was developed from the regorafenib-resistant GIST patient \#1. This PDX has a homozygous KIT exon 11 primary mutation (V559D) and a homozygous KIT exon 13 secondary ATP-binding pocket mutation (V654A). All in vivo work was conducted under appropriate Institutional Animal Care and Use-Committee-approved protocols. Six- to 8-week-old female adult athymic nude mice (NMRI nu/nu) were obtained from Charles River Laboratories (Wilmington, MA, USA) and housed under specific pathogen-free conditions. Tissue fragments of PG48 were serially passaged in donor mice injected subcutaneously in each rear flank. In all studies, vehicle control or study drugs were administered orally once daily. Solutions and drug doses were as follows: sterile water, and $100 \mathrm{mg} / \mathrm{kg} /$ day for Imatinib; citrate buffered $(\mathrm{pH} 3.5)$, and $40 \mathrm{mg} / \mathrm{kg} / \mathrm{day}$ for sunitinib $^{25}$; PEG400/125 mM aqueous methanesulphonic acid $(80 / 20)$, and $30 \mathrm{mg} / \mathrm{kg} /$ day for regorafenib. ${ }^{15}$ The experiment was stopped after 3 days of treatment, mice were sacrificed, and tumours were harvested for protein analysis.

Drug-withdrawal studies

GIST cell lines were cultured in serum-containing media in the presence of DMSO, imatinib, sunitinib or regorafenib at the indicated concentrations. Drugs were withdrawn and washed out, and cells were grown in regular media. Study time-points were as follows: day 0 (on drug), and days 1, 3 and 7 after drug withdrawal. Cultures were performed in 6-well plates to obtain cell lysates to assess KIT pathway and cell cycle activation, and in Lab-TEK II chamber slides (Thermo Fisher, Walthman, MA, USA; \#154526) to assess cell proliferation (KI67 immunostaining and mitotic count) as described above.

Validation of a rapid-alternation schedule in mixed GIST cell cultures

Human GIST cells with clinically representative KIT mutations (GIST-T1, GIST430/654 and GIST-T1/820) were stably infected with 
lentiviruses containing specific 24-basepair DNA "barcodes", as described previously. ${ }^{26}$ Equally numbers of each of the three cell lines were added to create mixed GIST cell cultures. Mixed GIST cell cultures were treated with sunitinib $(200 \mathrm{nM})$ and regorafenib $(400 \mathrm{nM})$, as single-agents or in rapid-alternation. Genomic DNA was isolated using QIAamp DNA Mini Kit (Quiagen, Germantown, MD, USA) on day 0 (baseline), 7, 14, 21 and 28, and analysed using PRISM technology. ${ }^{27}$ PRISM allows assessment of relative cell viability in mixtures of cell lines via the quantification of unique DNA barcodes incorporated into individual cell lines. Results are shown as percentage of cell numbers relative to untreated. Twosided unpaired t-test is used for cell number comparisons between treatment conditions.

\section{RESULTS}

Each of nine small molecule KIT-inhibitors has activity against only a subset of common imatinib-resistance oncogenic KIT mutations Nine TKIs that have either been approved or are under clinical investigation as KIT-inhibitors for GIST were evaluated by viability assays in GIST cell lines representing four biologic categories: 1) imatinib-sensitive with KIT primary mutations; 2 ) imatinib-resistant due to KIT ATP-binding pocket secondary mutations; 3 ) imatinibresistant due to KIT activation loop secondary mutations; and 4) imatinib-resistant with acquired loss of KIT oncoprotein expression (acquired KIT-negative). Each of these TKIs was active against GIST cells with KIT primary mutations in exon 11 (GIST-T1 and GIST430) but failed to inhibit the full range of secondary imatinibresistance mutations (Table 1 ). Imatinib and masitinib were ineffective against all of the common KIT secondary mutations. The other seven TKIs had activity against GIST cells with some but not all imatinib-resistance mutations (Table 1). Sunitinib and dovitinib inhibited viability of GIST cells dependent on the KIT exon 13 V654A secondary mutation, with $I_{50}$ values lower than $500 \mathrm{nM}\left(\mathrm{IC}_{50} 45 \mathrm{nM}\right.$ and $250 \mathrm{nM}$, respectively). The ponatinib IC 50 for GIST with KIT V654A was $100 \mathrm{nM}$, but this exceeds the clinically relevant concentration $(<28 \mathrm{nM})$ for this drug, ${ }^{28}$ confirming previous evidence that ponatinib is not sufficiently active against this common resistance mutation. ${ }^{19}$ A broader group of TKIs (sunitinib, regorafenib, sorafenib, ponatinib and dovitinib) inhibited GIST cells dependent on the "gatekeeper" KIT exon 14 T670I mutation ( $\left(\mathrm{C}_{50} 5-200 \mathrm{nM}\right)$. Sunitinib and dovitinib lacked activity against secondary mutations in the KIT activation loop (KIT exons 17 and 18), in contrast to regorafenib, sorafenib, nilotinib, ponatinib and dasatinib, which inhibited viability of GIST cells dependent on some or all of the activation-loop mutants (Table 1). No substantial TKI effects were observed in KIT-independent GIST cell lines GIST48B and GIST226 (Table 1), which underscores that TKI-activity is typically mediated by blocking KIT signalling in imatinib-resistant GIST. These observations are consistent with previously reported clinicopathologic evidence that short-duration clinical responses in KIT-mutant GIST, after development of imatinib-resistance, result from outgrowth of cross-resistant GIST subpopulations with both ATP-binding pocket and activation loop KIT secondary mutations. ${ }^{8-11}$

Regorafenib activity in GIST is mediated through KIT oncogenic signalling inhibition and displays a complementary activity profile with sunitinib against KIT secondary mutations

Evaluations of the three FDA- and EMA-approved drugs for the treatment of GIST, imatinib, sunitinib and regorafenib, demonstrated that each drug inhibited oncogenic KIT and downstream AKT phosphorylation in a dose-dependent manner in GIST cells containing only KIT primary exon 11 mutations (GIST-T1, GIST430 and GIST882) without KIT secondary mutations (Fig. 1a). By contrast, in GIST cells with KIT exon 11 primary mutations coupled to common secondary KIT imatinib-resistance mutations, KIT and downstream signalling were variably inhibited by

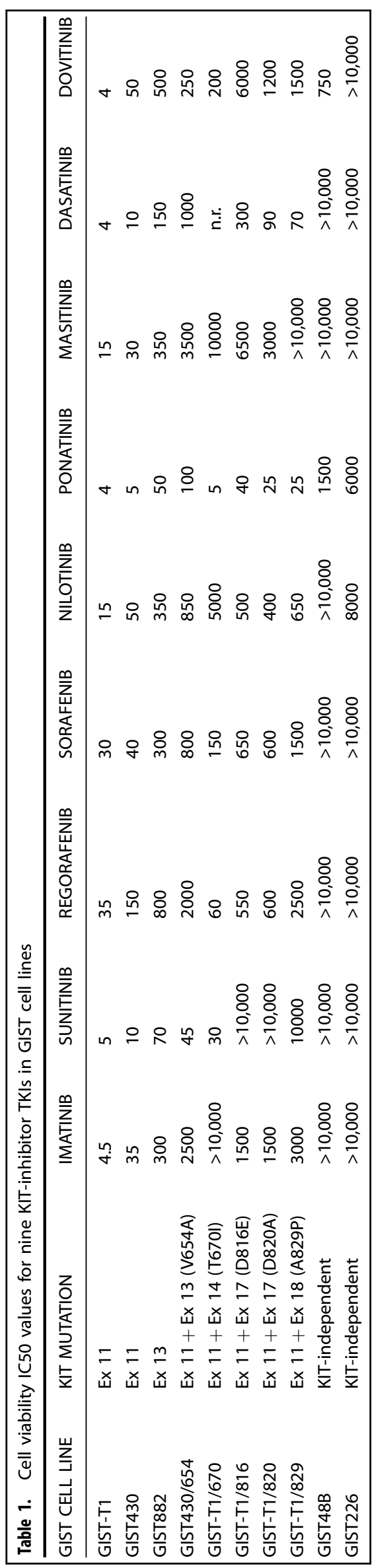


a

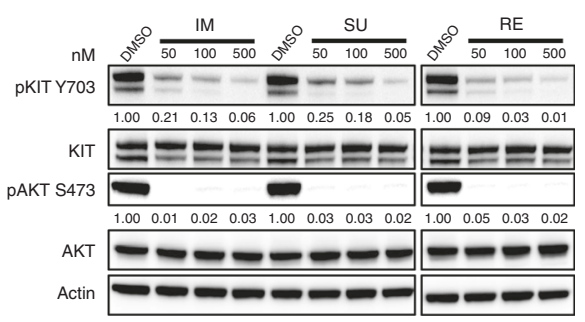

b

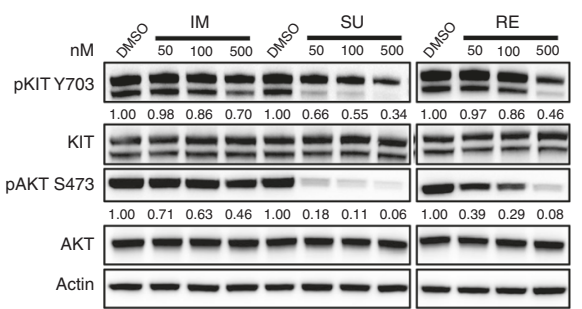

C

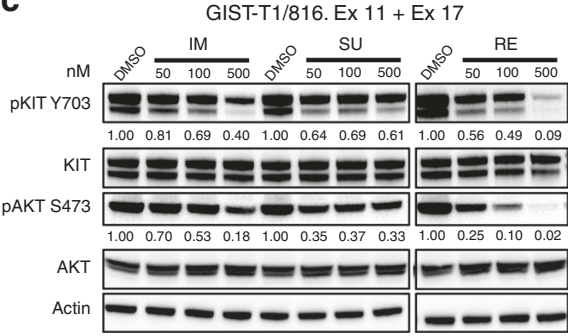

d

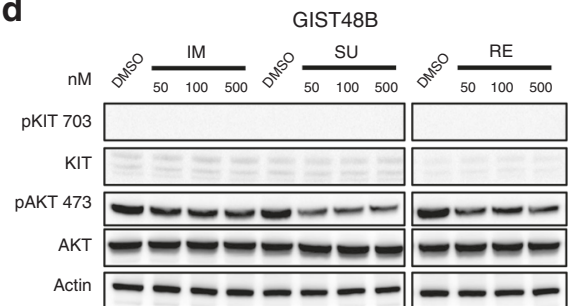

GIST430. Ex 11

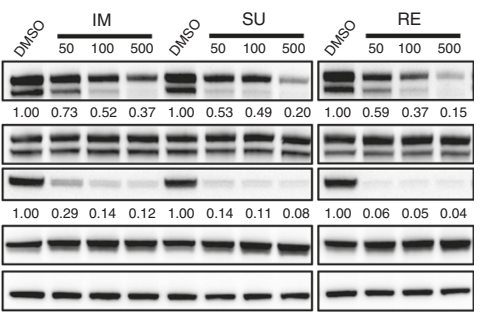

GIST-T1/670. Ex $11+$ Ex 14

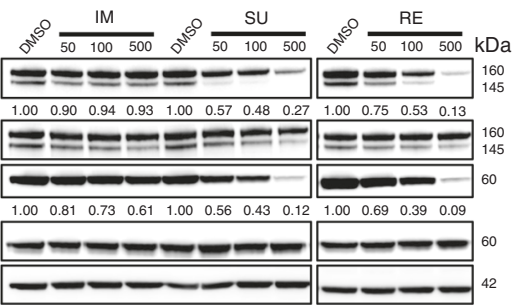

GIST-T1/820. Ex $11+$ Ex 17

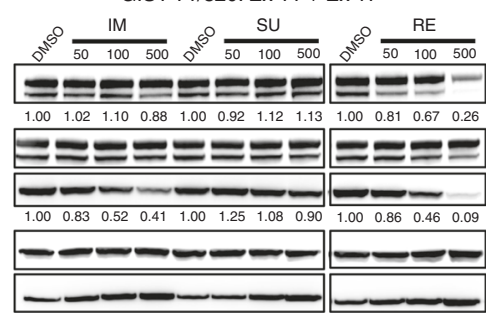

GIST226

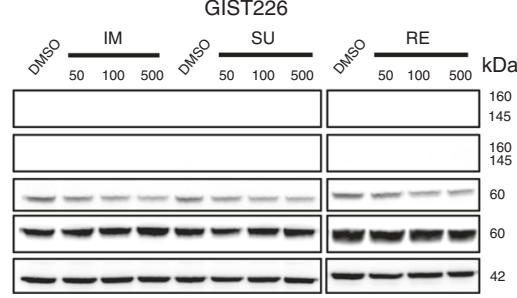

GIST882. Ex 13

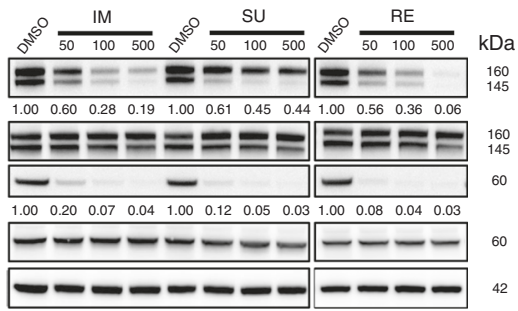

Fig. 1 KIT oncoproteins in GIST are differentially inhibited by sunitinib and regorafenib. Immunoblotting evaluations of phospho-KIT and downstream phospho-AKT were performed in GISTs belonging to four clinical-genotypic categories: (a) imatinib-sensitive GISTs contained only KIT primary mutations; (b) imatinib-resistant GISTs with ATP-binding pocket KIT secondary mutations; (c) imatinib-resistant GISTs with activation loop KIT secondary mutations; (d) KIT-negative GISTs. This figure also provides quantifications, relative to the DMSO-only controls (normalised to 1.0), of the (a-c) phosphoKIT and phosphoAKT responses in KIT-dependent GISTs

sunitinib and regorafenib. Specifically, GIST cells in which KIT exon 11 primary mutation was coupled to a secondary mutation in exon 13 V654A mutation were inhibited only by sunitinib, whereas GIST cells with KIT exon 11 primary mutation coupled to an exon 14 T670l gatekeeper mutation were inhibited by sunitinib and regorafenib (Fig. 1b), and GIST cells with KIT exon 11 primary mutation coupled to exon 17 D816E or D820A or exon 18 A829P activation loop mutations were inhibited by regorafenib only (Fig. 1c). Neither sunitinib nor regorafenib inhibited viability or AKT phosphorylation in KIT-negative GIST cell lines GIST48B and GIST226 (Fig. 1d). Consistent with these in vitro results in GIST cells, evaluations of primary and secondary KIT mutants transfected in Chinese Hamster Ovarian (CHO) cells showed that sunitinib was more active than regorafenib against the KIT exon 13 V654A ATP-binding pocket mutant, whereas regorafenib was more active than sunitinib against KIT exon 17 activation loop mutants (Supplementary Figure 1).
Correlative science studies show suboptimal regorafenib clinical activity against the common KIT V654A imatinib-resistance mutation in GIST

Imaging studies and tissue biopsies were obtained from two GIST patients treated with regorafenib in a phase II clinical trial. ${ }^{23}$ These two patients received standard regorafenib dosing (160 mg, 3-weeks on, 1-week off) throughout the study. After 9 months on therapy, patient \#1 developed a metabolically active mediastinal lesion (Fig. 2a), which was resected. Histologically, the lesion lacked treatment effect, and genotype analysis revealed a primary KIT exon 11 mutation and a secondary KIT exon 13 V654A mutation. The patient resumed regorafenib treatment and, after 12 months, developed further resistant disease in the abdomen; analysis of this lesion again revealed a KIT exon 13 V654A mutation, in addition to the primary KIT exon 11 mutation. By contrast, patient \#2 had pretreatment biopsy demonstrating primary KIT exon 11 and secondary D820Y (exon 17) mutations and achieved complete 
a
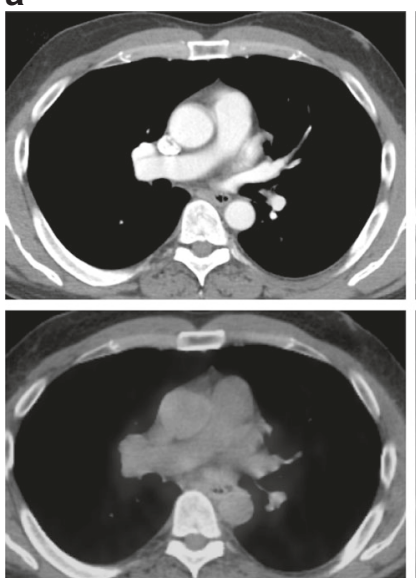
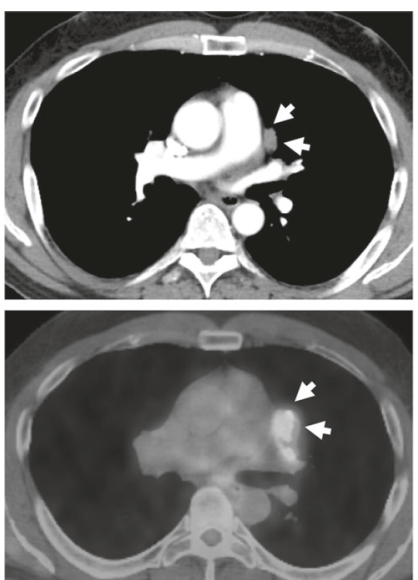

b
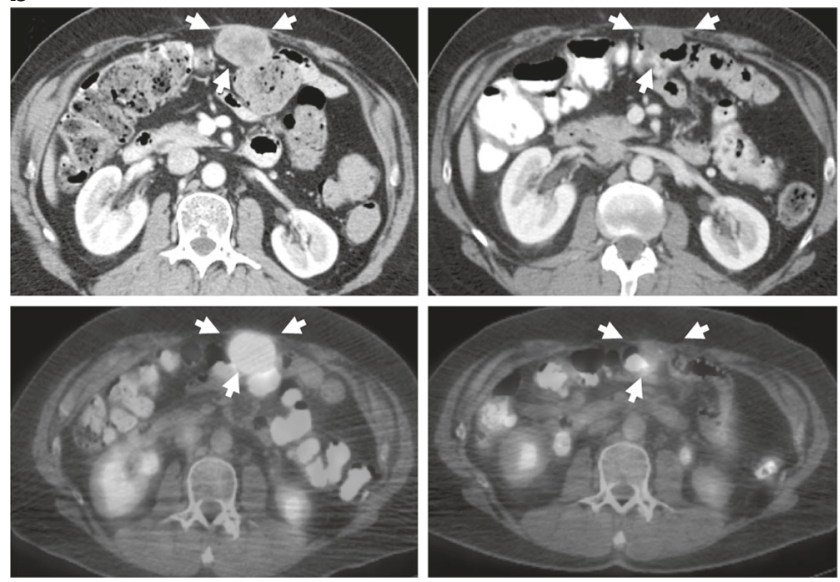

Fig. 2 Clinical evidence that GIST imatinib-resistant KIT mutations have differential responsiveness to regorafenib. Two GIST patients were treated at the standard regorafenib dose $(160 \mathrm{mg} / \mathrm{d}, 3$ weeks on, 1 week off) and imaging and tissue specimens were available. a Patent \#1 developed a new site of metastatic disease after 12 cycles of treatment, which was FDG-avid in the PET/CT. Resection and sequencing of this progressing lesion showed a V654A KIT ATP-binding pocket secondary mutation. b Patient \#2 had a $4.5 \times 3.2 \mathrm{~cm}$ metastatic lesion in the abdominal wall that was biopsied and sequenced prior to regorafenib therapy. A KIT activation-loop imatinibresistance mutation in exon 17 (D820Y) was found. After 5.5 months on regorafenib treatment, tumour size diminished to $3.0 \times 1.6 \mathrm{~cm}$ and SUV values decreased from 9.9 to $<3$

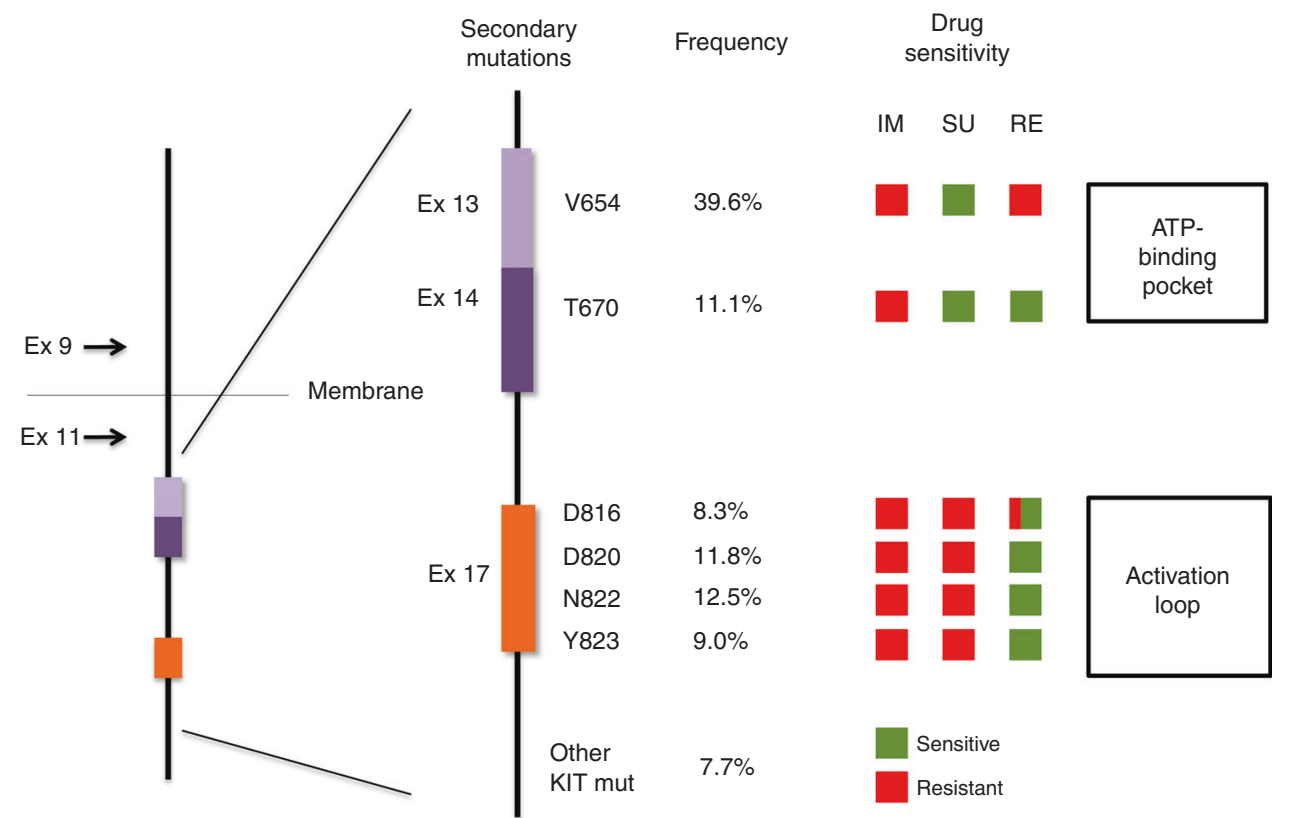

Fig. 3 Schematic view of representative KIT secondary mutations after imatinib failure, frequency according to prior reports, $8,9,11,18,36,37$ and predicted activity profile of the three drugs currently approved for the treatment of GIST based on our studies. Green and red denotes sensitive and resistant, respectively, to imatinib (IM), sunitinib (SU) and regorafenib (RE). Regorafenib square for D816 is both red and green due to the presence of resistant amino acid changes (i.e., D816V is highly resistant to all TKIs)

metabolic response and partial response by RECIST after 4 cycles of treatment with regorafenib (Fig. 2b). Complete metabolic response in this lesion was seen 18 days after treatment initiation.

We extended these in vitro and clinical data in a xenograft model derived from a regorafenib-resistant GIST, which contained KIT exon 11 and KIT exon 13 (V654A) mutations. In this model, KIT phosphorylation was inhibited substantially $(80 \%)$ by sunitinib treatment, but not $(8 \%)$ by regorafenib treatment (Supplementary Figure 2).
Proof-of-concept of rapid TKI alternation treatment in heterogeneous GIST co-cultures

Because the above-mentioned in vitro, in vivo and clinical data indicated complementary activity of sunitinib and regorafenib against the most common KIT imatinib-resistance secondary mutations (Fig. 3), we hypothesised that combined treatment with sunitinib and regorafenib might suppress a broad range of resistant subclones in GIST patients progressing on imatinib. However, sunitinib and regorafenib share overlapping toxic effects, and concurrent treatment with these drugs would likely 
a

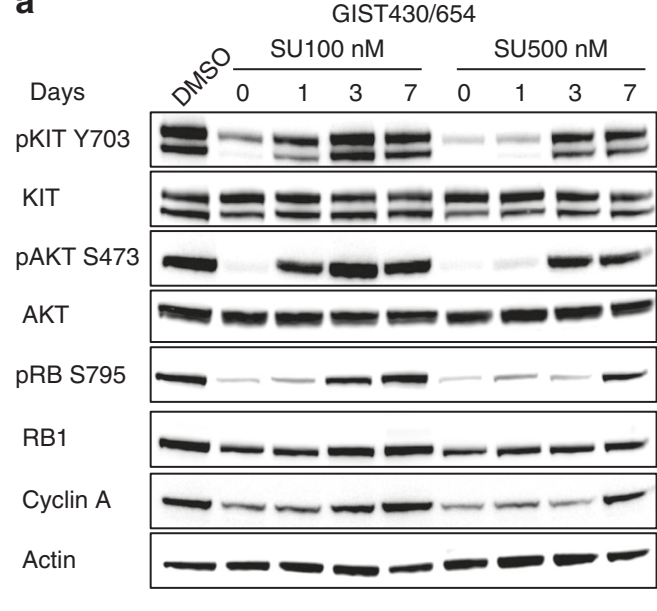

b

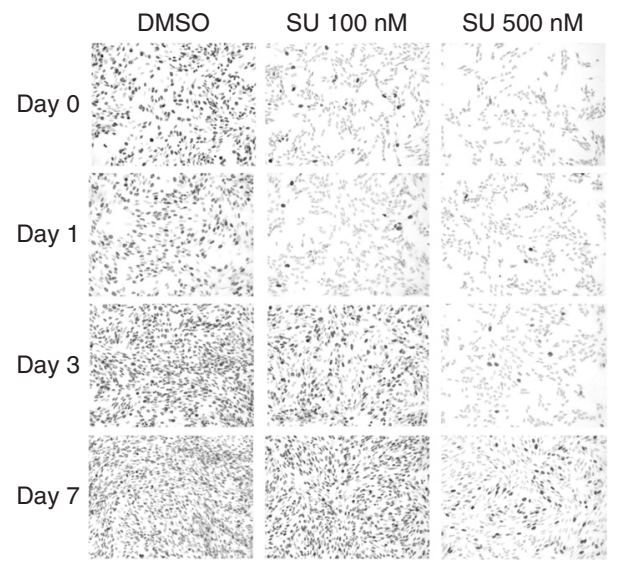

C

\begin{tabular}{|l|c|c|c|}
\hline \multicolumn{4}{|c|}{ Mitotic count (per 5 mm²) } \\
\hline & DMSO & $100 \mathrm{nM}$ & $500 \mathrm{nM}$ \\
\hline Day 0 & 62 & 4 & 1 \\
\hline Day 1 & 60 & 3 & 0 \\
\hline Day 3 & 63 & 45 & 1 \\
\hline Day 7 & 68 & 65 & 11 \\
\hline
\end{tabular}

GIST48/820
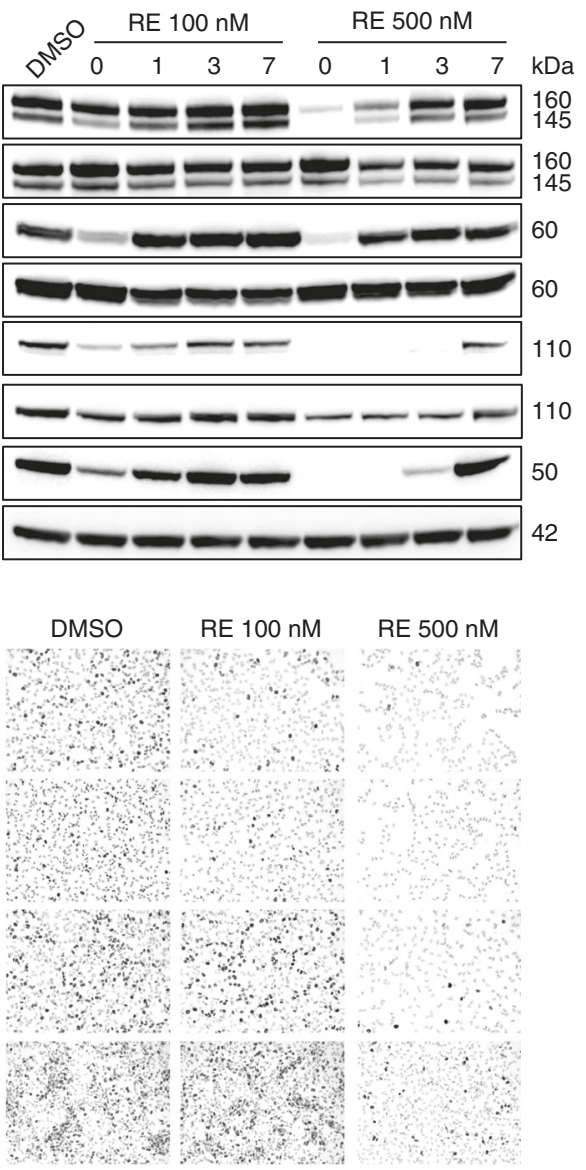

\begin{tabular}{|l|c|c|c|}
\hline \multicolumn{4}{|c|}{ Mitotic count (per 5 mm²) } \\
\hline & DMSO & $100 \mathrm{nM}$ & $500 \mathrm{nM}$ \\
\hline Day 0 & 42 & 15 & 1 \\
\hline Day 1 & 44 & 13 & 0 \\
\hline Day 3 & 54 & 63 & 2 \\
\hline Day 7 & 35 & 42 & 15 \\
\hline
\end{tabular}

Fig. 4 Restoration of GIST oncogenic signalling pathways and proliferation after TKI withdrawal. a Immunoblotting evaluations show reactivation of KIT and AKT, as assessed by phospho-KIT and phospho-AKT, and show reactivation of the cell cycle, as assessed by phosphoRB1 and Cyclin A. b Ki-67 staining shows recovery of proliferation after drug withdrawal. c, Mitotic counts (per $5 \mathrm{~mm}^{2}$ ) show recovery of proliferation after drug withdrawal

exacerbate each drug's toxicities. Therefore, we modelled a concept for rapid alternation of these drugs, aiming to inhibit growth of heterogeneous cross-resistant subclones, while minimising toxicity. Because this alternating dosing could permit regrowth of targeted subclones when the relevant drug for those subclones is withheld, we evaluated a rational time-frame for drug withdrawal, to establish a treatment schedule that minimises GIST regrowth during alternating drug withdrawal periods.

Drug withdrawal studies were performed using sunitinibsensitive GIST430/654 (Table 1) and regorafenib-sensitive GIST48/820, ${ }^{19}$ after treatment with sunitinib and regorafenib, respectively. In these studies, the drugs were withdrawn after inhibition of KIT phosphorylation and cell proliferation was achieved. Partial re-activation of KIT phosphorylation and downstream AKT phosphorylation was observed $24 \mathrm{~h}$ after drug withdrawal in both cell lines. Cell cycle re-activation, as evidenced by upregulation of phospho-RB1, cyclin A, and Ki-67, occurred 3-to- 7 days after drug withdrawal (Fig. 4a). Complete restoration of Ki-67 expression and mitotic activity occurred 7 days after drug withdrawal (Fig. $4 \mathrm{~b}$ and $\mathrm{c}$ ). These effects on KIT signalling and cell cycle re-activation were dose-dependent. These observations define a time-frame for TKI alternation in which substantial recovery of in vitro cell proliferation occurs within 7 days, whereas minimal, if any, recovery is seen within 3 days after TKI withdrawal.

To test the effects of drug withdrawal and rapid drug alternation on heterogeneous GIST populations, we established polyclonal co-cultures of GIST cell lines containing clinically relevant KIT mutations: exon 11 mutation alone (GIST-T1), exon 11 primary mutation in cis with exon 13 secondary mutation (GIST430/654), and exon 11 primary mutation in cis with exon 17 secondary mutation (GIST-T1/820), modelling the polyclonal imatinib-resistance heterogeneity observed in many GIST patients. Each cell line was labelled with a unique DNA barcode to allow quantification of the relative amount of each line in the co-culture 
a

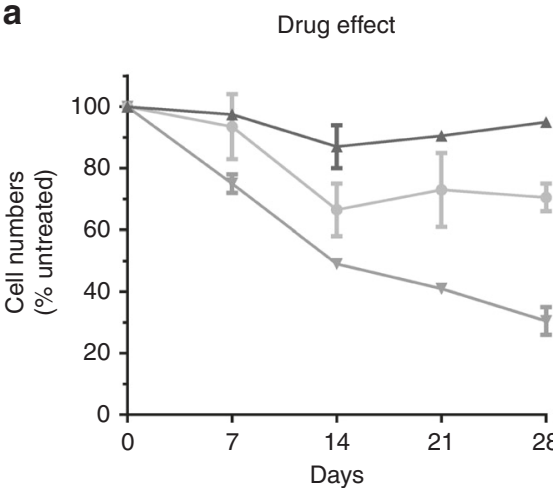

b

$$
\begin{aligned}
& \text { - SU } 200 \mathrm{nM} \\
& -\mathrm{RE} 400 \mathrm{nM}
\end{aligned}
$$$$
\rightarrow \text { Cycling }
$$

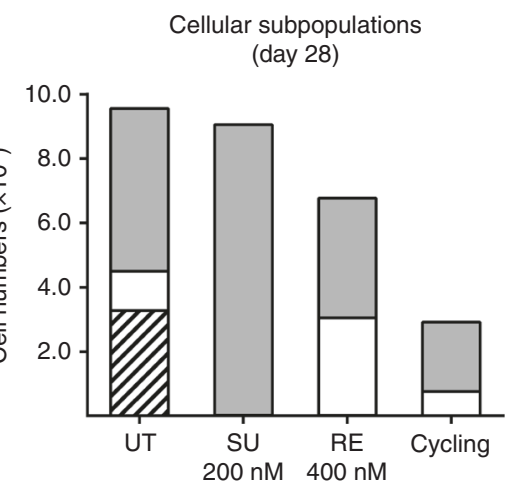

Fig. 5 Suppression of polyclonal imatinib-resistant populations by sunitinib and regorafenib rapid alternation. PRISM analysis of barcoded cell lines was used to assess mixed GIST cultures treated with single-agent sunitinib, single-agent regorafenib, or rapid alternation ( 3 days of sunitinib alternating with 4 days of regorafenib). a Cell numbers were lowest in the rapid alternation arm, at all time-points. b Population profiling at day 28 demonstrated partial suppression of ATP-binding-pocket (V654A) and activation loop (D820A) imatinib-resistant GIST cells by the rapid alternation approach

using the PRISM method. ${ }^{27}$ Co-cultures were established with equal representation of the three cell lines and expanded for 4 weeks under the following conditions: (1) untreated; (2) continuous sunitinib; (3) continuous regorafenib; or (4) rapid alternation of sunitinib and regorafenib (sunitinib 3 days, alternating with regorafenib 4 days). The sunitinib and regorafenib alternation was more effective than either drug alone with respect to inhibiting growth of these polyclonal populations and preventing emergence of a single dominant clone (Fig. 5a). In the GIST polyclonal co-cultures receiving monotherapies, we observed overrepresentation of the known cross-resistant clone. Although imatinib-resistant co-cultures were not eradicated, total numbers of both GIST-T1/820 and GIST430/654 were reduced in the co-cultures treated with rapid alternating combination of sunitinib and regorafenib (Fig. 5b).

\section{DISCUSSION}

The crucial oncogenic events in most GISTs are gain-of-function KIT mutations, which cause KIT tyrosine kinase constitutive activation and resultant constitutive signalling through growthpromoting pathways, including PI3K/AKT/mTOR and RAS/RAF/ MEK. ${ }^{29}$ These primary KIT mutations typically involve KIT exons 9 or 11, corresponding to extracellular dimerization domains and intracellular inhibitory juxtamembrane domains, respectively. The imatinib-resistance mechanisms responsible for clinical progression in KIT-mutant GISTs commonly involve emergence of polyclonal subpopulations with secondary KIT kinase domain mutations. ${ }^{8-11}$ KIT-independent mechanisms leading to imatinibprogression in KIT-mutant GIST have been reported but are infrequent and yet to be thoroughly studied. ${ }^{17,30}$ Therefore, KIT inhibitory strategies after failure of imatinib remain useful because most imatinib-resistant GISTs are still dependent upon KIT signalling for survival and proliferation. This has been evidenced by GIST antiproliferative responses in both preclinical models and patients, after treatment of imatinib-resistant GISTs by various KITinhibitor strategies, including RNA interference, ${ }^{9}$ alternative KIT kinase inhibitors such as sunitinib, ${ }^{12,18}$ or HSP90 inhibitors. ${ }^{31}$ However, the frequently polyclonal nature of GIST imatinibresistance constitutes a clinical challenge and explains why sunitinib, the current second-line standard-of-care, generally has only modest efficacy in GIST patients. ${ }^{14}$ Conceivably, this heterogeneous nature of KIT secondary mutations also limits the clinical benefit of subsequent lines of treatment, including thirdline regorafenib ${ }^{13}$ and other TKIs under investigation, such that median time to progression is in the same range as that with sunitinib. ${ }^{16,17}$
We and others have previously demonstrated that sunitinib clinical activity for most GISTs is determined by the primary and secondary KIT mutations in those tumours. ${ }^{12,18}$ In the present study, we provide the first evidence of regorafenib spectrum of inhibition of imatinib-resistant KIT mutations. We profiled regorafenib activity against a panel of GIST cell lines containing the common types of KIT imatinib-resistance secondary mutations in GIST, involving either the KIT ATP-binding pocket or activation loop. Regorafenib, like imatinib and sunitinib, had compelling activity against GIST models dependent only on KIT exon 11 primary mutations, i.e. lacking imatinib-resistant secondary KIT mutations. In addition, regorafenib inhibited KIT kinase activation loop secondary imatinib-resistance mutations, which are known to be sunitinib resistant. ${ }^{12,18}$ The evidence for regorafenib activity against KIT secondary activation loop mutations included preclinical modelling of drug effects on cell proliferation, KIT phosphorylation, and KIT-pathway phosphorylation. Further, we document regorafenib clinical response in a patient whose GIST had a KIT activation loop imatinib-resistance mutation (Fig. 2b), supporting evidence we reported previously. ${ }^{23}$ Nonetheless, KIT activation loop secondary mutations are heterogeneous and the present study demonstrates variable regorafenib activity depending on the exact activation loop amino acid alteration. For example, regorafenib was more active against KIT exon 17 D816E mutation than D816H. Other KIT oncogenic mutations, including an exon $13 \mathrm{~K} 642 \mathrm{E}$ primary mutation, and the exon 14 T670l "gatekeeper" secondary mutation, were inhibited effectively by both sunitinib and regorafenib. $^{32,33}$ Very similar GIST inhibition profiles were demonstrated for regorafenib and the structurally-related compound, sorafenib, demonstrating the reproducibility of the models.

Regorafenib had only modest activity against the KIT exon 13 V654A secondary mutation, which modifies a residue in the ATPbinding pocket of the kinase and is the most common secondary imatinib-resistance mutation in GIST patients following primary treatment with imatinib. ${ }^{18}$ In the present study, a patient progressed twice on a standard regorafenib dose/schedule, and on each occasion the regorafenib-resistance resulted from KIT V654A secondary mutation. Likewise, in preclinical studies, we demonstrated modest inhibition of KIT V654A phosphorylation only at high doses of regorafenib. Therefore, the evidence to date indicates that regorafenib does not inhibit effectively the KIT V654A imatinib-resistance mutation in GIST. By contrast, KIT V654A is inhibited effectively by sunitinib, as shown in our present evaluations and in previously published preclinical and clinical studies. $^{12,18}$ 
Our cell viability studies using 9 different TKIs, either approved or under clinical investigation in GIST, underscore that each of the targeted KIT-inhibitor small molecule drugs included in this analysis is active against only a subset of the common imatinibresistance secondary KIT mutations. As discussed above, these limitations have been established in previous studies for sunitinib, ${ }^{12,18}$ and are now reported, herein, for regorafenib. Likewise, our current studies confirm that ponatinib inhibits some but not all common imatinib-resistance secondary KIT mutations, with the common KIT exon 13 V654A mutation being a particular challenge. ${ }^{19}$ Although the drug screens show differential activity for each of the TKIs against various imatinib-resistance KIT mutations, the in vitro $\mathrm{IC}_{50}$ values do not permit precise prediction of clinical activity, particularly in absence of correlative studies from GIST biopsies and/or cfDNA in patients receiving these agents. Overall, regorafenib and sorafenib had comparable activity in vitro. Although sorafenib $\mathrm{IC}_{50}$ for KIT exon $13 \mathrm{~V} 654 \mathrm{~A}$ mutation appears to be lower compared to regorafenib ( $800 \mathrm{nM}$ vs. 2000 $\mathrm{nM})$, we do not expect meaningful clinical differences for these two drugs. Masitinib activity fully overlaps with imatinib, showing a >10-100-fold gap between $\mathrm{IC}_{50}$ in imatinib-sensitive vs. imatinib-resistant mutants. Nilotinib and dasatinib have similar profiles, with activity in imatinib-sensitive models and potential activity against activation-loop mutants, which agrees with previous reports. ${ }^{34,35}$ However, and in the light of published clinical data in imatinib-resistant disease, it is unlikely that nilotinib and dasatinib activity against KIT secondary mutations in the activation loop is comparable to that of regorafenib or ponatinib. ${ }^{16,17}$ Finally, dovitinib activity appeared to be comparable to sunitinib in our studies, with the $\mathrm{IC}_{50}$ for ATP-binding pocket mutations being 6-30-fold lower than those for mutations in the activation-loop. All studies in KIT-independent cell lines confirm that the activity of these TKIs is mediated through KIT oncogenic signalling inhibition. Together, these studies reinforce that KIT secondary genotype determines the activity of TKIs with KIT inhibitory activity in GIST, thereby establishing the molecular basis for the modest clinical benefit observed with successive lines of treatment in imatinib-resistant GIST.

The incomplete coverage of imatinib-resistant KIT mutations by each of these drugs allows cross-resistant GIST subclones to emerge from a background of heterogeneous GIST cells with varied KIT secondary mutations, leading to clinical progression irrespective of the TKI used. Therefore, more effective strategies for KIT-inhibition are needed in patients with imatinib-resistant GIST, in order to accomplish longer-lasting clinical benefit. Hence, we investigated whether rational combinations of KIT inhibitors could overcome polyclonal resistance after imatinib failure. Notably, several drugs from our screening displayed complementary activity profiles with respect to inhibiting either the KIT exon 13 V654A mutation or KIT exon 17 activation loop mutations. Each of these mutation categories accounts for approximately $40 \%$ of imatinib-resistant subclones in GIST patients (Fig. 3). ${ }^{8-10,18,36,37}$ In order to enable translation into a near-term clinical trial in GIST patients, we focused in these preclinical studies on novel approaches to administering sunitinib and regorafenib, the two currently approved drugs for imatinib-resistant GIST. Therapeutic combinations may augment the magnitude and/or duration of clinical responses, ${ }^{38,39}$ and the observed complementary pattern suggests that a combination of sunitinib and regorafenib could broaden the spectrum of subclones effectively targeted by second-line therapy, delaying the emergence of cross-resistant disease and extending the window of clinical benefit beyond the 4-6 months currently observed with each drug as singleagent. ${ }^{13,14}$ However, both drugs share overlapping toxicities and concurrent treatment would require dose reductions leading to loss of efficacy in order to minimise adverse events. ${ }^{38,39}$ Several studies have shown that continuous dosing is not necessary for drug efficacy, ${ }^{40-44}$ and might decrease selective pressure and minimise positive subclonal selection and subsequent disease progression: ${ }^{41,42}$ these observations beg the question as to whether sequentially alternating sunitinib and regorafenib could achieve effective doses of both drugs while minimising toxicity. Thus, we identified and validated in GIST cell models a concept for rapid-alternation of sunitinib and regorafenib, aiming to maintain inhibitory pressure on the two major types of imatinib-resistance KIT mutations that coexist in many GIST patients. These data provide rationale for a clinical trial in which alternation of sunitinib for 3 days with regorafenib for 4 days is currently being evaluated in GIST patients with advanced disease (ClinicalTrials identifier: NCT02164240).

In summary, this study defines the spectrum of inhibition of imatinib-resistant KIT mutations by third-line regorafenib and multiple available TKIs. We find that small molecule KIT-inhibitor monotherapies have drug-specific activity profiles against only subsets of the KIT secondary imatinib-resistance mutations, which constitutes the molecular basis for the modest clinical benefit observed with successive lines of treatment in imatinib-resistant GIST. Our studies underscore the need for therapies that suppress the spectrum of heterogeneous imatinib-resistant subclones that can arise in a given GIST patient. Leveraging these insights, we show that rapid alternation of TKIs with complementary activity can control heterogeneous imatinib-resistance subclones.

\section{AUTHOR CONTRIBUTIONS}

Conception and/or design: All authors. Collection, generation and assembly of data: C.S., A.M.E., D.L.T., J.K., G.E., M.Z., C.Y., A.M.M., A.P., A.M., J.T.C., Contribution with reagents/tools: C.Y., A.M.M., B.P.R., C.P.R., G.D.D., M.C.H., E.S., S.B., S.G., J.A.F. Manuscript writing: C.S., S.G. and J.A.F. Manuscript revision: All authors. Final manuscript approval: All authors. Agree to be accountable for all aspects of the work: All authors.

\section{ADDITIONAL INFORMATION}

Supplementary information is available for this paper at https://doi.org/10.1038/ s41416-019-0389-6.

Competing interests: The authors declare no competing interests.

Data availability: All the conclusions reached by the authors are supported by available main and supplementary tables and figures.

Ethics approval and consent to participate: GIST patients' samples were obtained for these studies from the phase II trial of single-agent regorafenib in GIST. ${ }^{23}$ The study was conducted in accordance with the ethical principles originating from the Declaration of Helsinki and Good Clinical Practice. All patients provided written informed consent before enrolment onto the study. The protocol was reviewed and approved by the institutional review board at all participating sites. PDX experiments were undertaken as previously described. ${ }^{45}$ All procedures were carried out according to protocols approved by the Institutional Animal Care and Use Committee of the Dana-Farber Cancer Institute.

Funding: This work was supported in part by an ASCO Young Investigator Award (CS), a Spanish Society of Medical Oncology Translational Award (CS), Río HortegaISCIII CM14/00241 (CS) FERO Foundation (CS), US National Institutes of Health grants 1P50CA127003 (GDD, ES, JAF), 1P50CA168512 (JAF, AME), GIST Cancer Research Fund (JAF, MCH), Life Raft Group (JAF, MCH, SB), V Foundation Translational Grant $(\mathrm{MCH})$, VA Merit Review Award (2101BX000338-05) $(\mathrm{MCH})$ and the Deutsche Krebshilfe (SB). CS acknowledges to the Cellex Foundation for providing facilities and equipment.

Note: This work is published under the standard license to publish agreement. After 12 months the work will become freely available and the license terms will switch to a Creative Commons Attribution 4.0 International (CC BY 4.0).

Publisher's note: Springer Nature remains neutral with regard to jurisdictional claims in published maps and institutional affiliations. 


\section{REFERENCES}

1. Demetri, G. D. et al. NCCN Task Force report: update on the management of patients with gastrointestinal stromal tumors. J. Natl. Compr. Cancer Netw.: JNCCN 8(Suppl 2), S1-S41 (2010).

2. Ducimetiere, F. et al. Incidence of sarcoma histotypes and molecular subtypes in a prospective epidemiological study with central pathology review and molecular testing. PLOS ONE 6, e20294 (2011).

3. Heinrich, M. C. et al. PDGFRA activating mutations in gastrointestinal stromal tumors. Science 299, 708-710 (2003)

4. Hirota, S. et al. Gain-of-function mutations of c-kit in human gastrointestinal stromal tumors. Science 279, 577-580 (1998).

5. Tuveson, D. A. et al. STI571 inactivation of the gastrointestinal stromal tumor cKIT oncoprotein: biological and clinical implications. Oncogene 20, 5054-5058 (2001).

6. Demetri, G. D. et al. Efficacy and safety of imatinib mesylate in advanced gastrointestinal stromal tumors. N. Engl. J. Med. 347, 472-480 (2002).

7. Blanke, C. D. et al. Long-term results from a randomized phase II trial of standardversus higher-dose imatinib mesylate for patients with unresectable or metastatic gastrointestinal stromal tumors expressing KIT. J. Clin. Oncol. 26, 620-625 (2008)

8. Desai, J. et al. Clonal evolution of resistance to imatinib in patients with metastatic gastrointestinal stromal tumors. Clin. Cancer Res. 13(18 Pt 1), 5398-5405 (2007).

9. Heinrich, M. C. et al. Molecular correlates of imatinib resistance in gastrointestinal stromal tumors. J. Clin. Oncol. 24, 4764-4774 (2006).

10. Liegl, B. et al. Heterogeneity of kinase inhibitor resistance mechanisms in GIST. J. Pathol. 216, 64-74 (2008).

11. Wardelmann, E. et al. Polyclonal evolution of multiple secondary KIT mutations in gastrointestinal stromal tumors under treatment with imatinib mesylate. Clin. Cancer Res. 12, 1743-1749 (2006).

12. Demetri, G. D. et al. Molecular target modulation, imaging, and clinical evaluation of gastrointestinal stromal tumor patients treated with sunitinib malate after imatinib failure. Clin. Cancer Res. 15, 5902-5909 (2009).

13. Demetri, G. D. et al. Efficacy and safety of regorafenib for advanced gastrointestinal stromal tumours after failure of imatinib and sunitinib (GRID): an international, multicentre, randomised, placebo-controlled, phase 3 trial. Lancet 381, 295-302 (2013).

14. Demetri, G. D. et al. Efficacy and safety of sunitinib in patients with advanced gastrointestinal stromal tumour after failure of imatinib: a randomised controlled trial. Lancet 368, 1329-1338 (2006).

15. Wilhelm, S. M. et al. Regorafenib (BAY 73-4506): a new oral multikinase inhibitor of angiogenic, stromal and oncogenic receptor tyrosine kinases with potent preclinical antitumor activity. Int. J. Cancer 129, 245-255 (2011).

16. Corless, C. L., Barnett, C. M. \& Heinrich, M. C. Gastrointestinal stromal tumours: origin and molecular oncology. Nat. Rev. Cancer 11, 865-878 (2011).

17. Serrano, C. \& George, S. Recent advances in the treatment of gastrointestinal stromal tumors. Ther. Adv. Med. Oncol. 6, 115-127 (2014).

18. Heinrich, M. C. et al. Primary and secondary kinase genotypes correlate with the biological and clinical activity of sunitinib in imatinib-resistant gastrointestinal stromal tumor. J. Clin. Oncol. 26, 5352-5359 (2008).

19. Garner, A. P. et al. Ponatinib inhibits polyclonal drug-resistant KIT oncoproteins and shows therapeutic potential in heavily pretreated gastrointestinal stromal tumor (GIST) patients. Clin. Cancer Res. 20, 5745-5755 (2014).

20. Rubin, B. P. et al. KIT activation is a ubiquitous feature of gastrointestinal stromal tumors. Cancer Res. 61, 8118-8121 (2001).

21. Wang, Y. et al. Dystrophin is a tumor suppressor in human cancers with myogenic programs. Nat. Genet. 46, 601-606 (2014)

22. Skehan, P. et al. New colorimetric cytotoxicity assay for anticancer-drug screening. J. Natl Cancer Inst. 82, 1107-1112 (1990). e-pub ahead of print 1990/07/04.

23. George, S. et al. Efficacy and safety of regorafenib in patients with metastatic and/or unresectable Gl stromal tumor after failure of imatinib and sunitinib: a multicenter phase II trial. J. Clin. Oncol. 30, 2401-2407 (2012). e-pub ahead of print 2012/05/23.
24. Eisenhauer, E. A. et al. New response evaluation criteria in solid tumours: revised RECIST guideline (version 1.1). Eur. J. Cancer 45, 228-247 (2009).

25. Mendel, D. B. et al. In vivo antitumor activity of SU11248, a novel tyrosine kinase inhibitor targeting vascular endothelial growth factor and platelet-derived growth factor receptors: determination of a pharmacokinetic/pharmacodynamic relationship. Clin. Cancer Res. 9, 327-337 (2003).

26. Ou, W. B., Zhu, M. J., Demetri, G. D., Fletcher, C. D. \& Fletcher, J. A. Protein kinase C-theta regulates $\mathrm{KIT}$ expression and proliferation in gastrointestinal stromal tumors. Oncogene 27, 5624-5634 (2008).

27. $\mathrm{Yu}, \mathrm{C}$. et al. High-throughput identification of genotype-specific cancer vulnerabilities in mixtures of barcoded tumor cell lines. Nat. Biotechnol. 34, 419-423 (2016).

28. Moslehi, J. J. \& Deininger, M. Tyrosine kinase inhibitor-associated cardiovascular toxicity in chronic myeloid leukemia. J. Clin. Oncol. 33, 4210-4218 (2015).

29. Bauer, S., Duensing, A., Demetri, G. D. \& Fletcher, J. A. KIT oncogenic signaling mechanisms in imatinib-resistant gastrointestinal stromal tumor: PI3-kinase/AKT is a crucial survival pathway. Oncogene 26, 7560-7568 (2007).

30. Hemming, M. L. et al. Gastrointestinal stromal tumor enhancers support a transcription factor network predictive of clinical outcome. Proc. Natl Acad. Sci. U. S. A. 115, E5746-E5755 (2018).

31. Bauer, S., Yu, L. K., Demetri, G. D. \& Fletcher, J. A. Heat shock protein 90 inhibition in imatinib-resistant gastrointestinal stromal tumor. Cancer Res. 66, 9153-9161 (2006).

32. Guo, T. et al. Sorafenib inhibits the imatinib-resistant KITT670I gatekeeper mutation in gastrointestinal stromal tumor. Clin. Cancer Res. 13, 4874-4881 (2007).

33. Heinrich, M. C. et al. Sorafenib inhibits many kinase mutations associated with drug-resistant gastrointestinal stromal tumors. Mol. Cancer Ther. 11, 1770-1780 (2012).

34. Cauchi, C. et al. Evaluation of nilotinib in advanced GIST previously treated with imatinib and sunitinib. Cancer Chemother. Pharmacol. 69, 977-982 (2012).

35. Schittenhelm, M. M. et al. Dasatinib (BMS-354825), a dual SRC/ABL kinase inhibitor, inhibits the kinase activity of wild-type, juxtamembrane, and activation loop mutant KIT isoforms associated with human malignancies. Cancer Res. 66, 473-481 (2006).

36. Antonescu, C. R. et al. Acquired resistance to imatinib in gastrointestinal stromal tumor occurs through secondary gene mutation. Clin. Cancer Res. 11, 4182-4190 (2005).

37. Debiec-Rychter, M. et al. Mechanisms of resistance to imatinib mesylate in gastrointestinal stromal tumors and activity of the PKC412 inhibitor against imatinibresistant mutants. Gastroenterology 128, 270-279 (2005).

38. Kummar, S. et al. Utilizing targeted cancer therapeutic agents in combination: novel approaches and urgent requirements. Nat. Rev. Drug. Discov. 9, 843-856 (2010).

39. Yap, T. A., Omlin, A. \& de Bono, J. S. Development of therapeutic combinations targeting major cancer signaling pathways. J. Clin. Oncol. 31, 1592-1605 (2013).

40. Abdel-Wahab, O. et al. Efficacy of intermittent combined RAF and MEK inhibition in a patient with concurrent BRAF- and NRAS-mutant malignancies. Cancer Discov. 4, 538-545 (2014).

41. Chmielecki, J. et al. Optimization of dosing for EGFR-mutant non-small cell lung cancer with evolutionary cancer modeling. Sci. Transl. Med. 3, 90ra59 (2011).

42. Das Thakur, M. et al. Modelling vemurafenib resistance in melanoma reveals a strategy to forestall drug resistance. Nature 494, 251-255 (2013).

43. Hoeflich, K. P. et al. Intermittent administration of MEK inhibitor GDC-0973 plus PI3K inhibitor GDC-0941 triggers robust apoptosis and tumor growth inhibition. Cancer Res. 72, 210-219 (2012).

44. Shah, N. P. et al. Transient potent BCR-ABL inhibition is sufficient to commit chronic myeloid leukemia cells irreversibly to apoptosis. Cancer Cell. 14, 485-493 (2008).

45. Nakayama, R. et al. Preclinical activity of selinexor, an inhibitor of XPO1, in sarcoma. Oncotarget 7, 16581-16592 (2016) 